\title{
A Simulated Model for Assesing the Line Condition of Onshore Pipelines
}

\author{
Shaik Nagoor Basha ${ }^{1, *}$, Pedapati Srinivasa Rao ${ }^{1}$ \\ ${ }^{1}$ Mechanical Engineering Department, Universiti Teknologi PETRONAS Bandar Seri Iskandar, \\ Perak, Malaysia
}

\begin{abstract}
Pipelines are considered safest mode of transport because of their limited number of facilities. It is therefore very important to monitor and optimize their operation and reduce their facilities to acceptable limits. Hence, it is an immediate requirement to assess and predict condition of existing oil and gas pipelines and to prioritize the planning of their inspection on a timely basis. Therefore, this study presents the development of models based on specific factors, that can predict the condition of onshore oil and gas pipelines. The model was developed using BPN (Back Propagation Network) techniques based on historical inspection data collected from the oil and gas fields. The model is expected to help pipeline operators to assess the condition of existing oil and gas pipelines and hence prioritize their inspection and rehabilitation operations.
\end{abstract}

\section{Introduction}

Pipelines are an important component in the distribution process of oil and natural gas. The integrity of these critical assets is heavily endangered by electrochemical deterioration which is usually referred as corrosion. The rate of corrosion is generally related to external and internal factors [1]. The pipeline networks are prolonged for a number of kilometers and consists of multiple pipe segments which are attached by joints or connections. Moreover pipelines are subjected to quite a few occurrences like traffic and external loads which leads to overstress on the pipes that can lead to leakage and burst in the pipeline [2]. So, for a well maintenance of pipeline there are some physical methods as well as computational methods to predict the condition of a pipeline. Soft computational techniques are used for the prediction purpose mostly. Out of all techniques some of the methods are used mostly like artificial neural networks, regression analysis, fuzzy logics etc. In the present research work, ANN (Artificial Neural Network) techniques are used for the development of models for forecasting the condition of pipeline.

The failure of a pipeline could be the outcome of many reasons like pressure differences, weld joints between the pipes, external stress and also due to aging. Abundant studies to detect and to know the condition of pipelines are introduced. Particle swarm optimization (PSO) technique was used by Liao et.al. for the prediction of corrosion rate internally by developing a model especially for wet gas gathering pipelines. Bersani et al. collected the inspectional data from the US Department of Transportation (DOT) in order to develop a risk

\footnotetext{
${ }^{*}$ Corresponding author: basha.roshan@gmail.com
} 
assessment model that can predict the disaster made by third party activity[3]. M. Bertolini et.al. used a tool based model to expect the period of each spillage in oil pipelines by means of statistical analysis classification and regression tree [4]. Fuzzy logic technique was used by Singh and Markeset to assess the corrosion rate by means of a risk based inspection program [5]. For detecting the faults due to corrosion and to measure the lasting life of pipeline, M. Ahammed presented a specific method [6]. Furthermore, El-Abbasy et al. built a model that can assess the state of oil $\&$ gas pipelines considering some factors using two different techniques such as Analytic Network Process (ANP) and Monte-Carlo simulation[7]. Later the model is tested on offshore gas pipeline located in Qatar which is already existed, and then the results obtained were compared with real conditions. El-Abbasy et al. gathered data of 3 dissimilar pipelines of different sizes (i.e. diameters), materials, and product type [8]. The parameters such as "anode wastage", "support condition", "joint condition", and "free spans", considered additionally in another study. The developed models were validated and the reults showed $96 \%$ accuracy. This study also provided a scale assessment for knowing the surrent status of pipeline which would be very much useful for the tecnicians in future . K. Mollazade et al. related few different techniques such as ANN, SVM, DT, and BN [9]. The results of validation showed that ANN had the maximum accuracy, i.e. $96.33 \%$.

In the present study, an attempt has been made to develop a simulated BPN model for assessing the line condition of oil and gas pipelines.

\section{Methodology}

The proposed methodology was started by gathering an inclusive data collection, with the investigation of the factors that are effecting the oil \& gas pipeline and factors that are required to rise the efficiency of the condition prediction procedure based on previous inspection records. However, it was concerned on specific parameters that are effecting the pipeline condition since the last decades. After considering specific parameters, attempts were made to eliminate or reduce the effect of those factors on pipeline by implementing ANN techniques for the safety of pipeline which will be the great use for the operators to predict the pipeline condition with in a span of less time. Next, the model was tested after the training and development of model was completed, later the model is tested and allowed for the validation process. Finally, the results were compared with actual ones i.e. graphs were plotted for actual vs predicted outputs.

\subsection{Factors that are considered for the development of BPN model}

There are numerous factors that can be taken in to consideration for the model development which has influence on pipelines, as the data is limited from the source, only few factors were focused as per data available. The below are the parameters that were considered which are affecting the onshore pipeline and those are as follows

- Metal loss

- Pressure flow

- Weld girth

- Cathode protection

\subsection{ANN architecture with specific fators as inputs}


The inputs were given from the historical data of a pipeline as shown in the Fig.1. and the model was trained based on algorithm written until the training standards were met. After the completion of training stage, it was tested for different testing input values. The testing input data sets were familiarized to the model that was trained in order to produce the expected output, and it was related to the actual one. Upon achieving accuracy of $>95 \%$ for predicted output, the model testing was classified as successful.

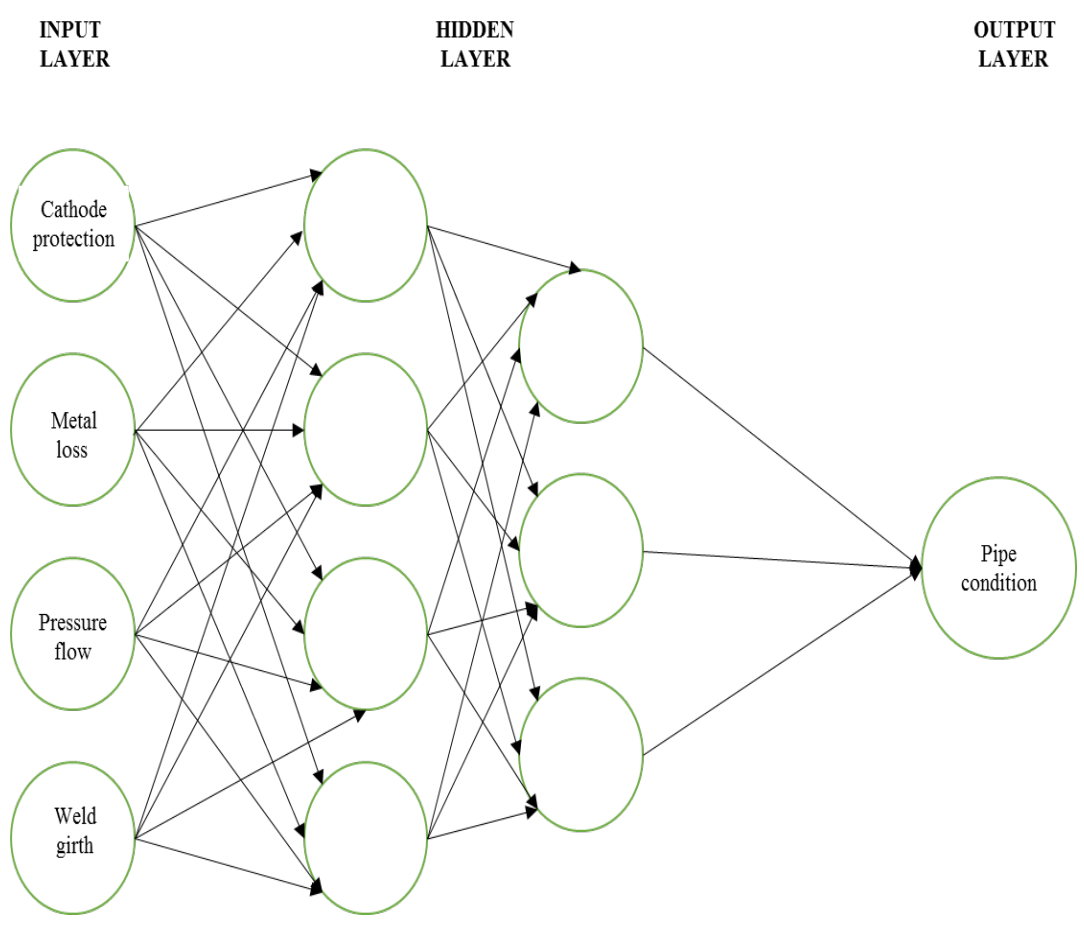

Fig. 1. ANN Architecture with specific inputs.

\subsection{Typical stages involved in ANN:}

\subsubsection{Training:}

In this stage, selection of learning algorithm is identified and the model is developed.

\subsubsection{Testing:}

The model is tested for different various cases in this stage.

\subsubsection{Validation:}

Finally, the model is validated for various responses which would be useful for the development of deterioration curves and these results would be helpful for the operators for the further assessment. 


\section{Results}

The BPN model was trained based on sigmoidal function where the 400 inputs are taken from parameters of input data, i.e.

$$
U_{k}=\sum_{j=1}^{m} w_{k j} x_{j}
$$

A Sigmoidal function was coded, which is a learning algorithm for training of model, and the function is given below:

$$
W_{y}=\cos [\sin (2 \pi W x 1)] \times \cos [\sin (2 \pi W x 2)]
$$

After that, it was tested for different values to get the accurate results and finally graphs were plotted and compared i.e. the actual curve vs predicted curve
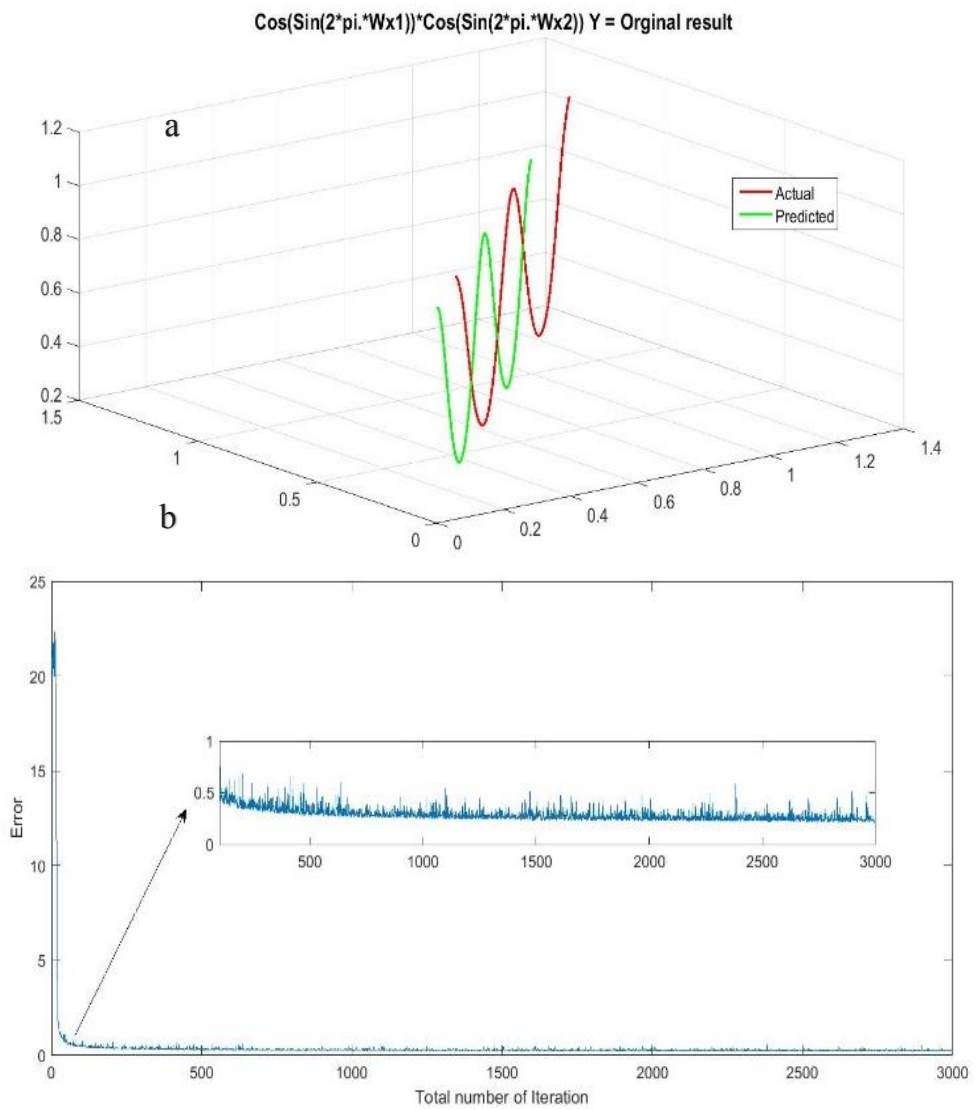

Fig. 2. Plots of (a) w shaped function i.e. actual versus predicted and (b) error for 3000 iterations.

In the above plots Fig.2. (a) actual vs predicted: which is similar to w-shaped function and (b)algorithm is run for 3000 iterations that shows minimum error. From these two plots, it can be stated that the results are accurate for simulated data, and the future work will be implemented on real time data.

\section{Conclusion}


This study presented development of model using BPN (Back Propagation Network) technique for the prediction of oil \& gas pipeline centered on specific parameters. The models were estimated to support pipeline operators to assess and forecast the condition of existing pipelines. The model was trained based on sigmoidal function until the training crieteria met and tested for different cases, finally the graphs were plotted which shows the accuracy and minimum error for simulated data. This work will be implemented on real time data in future.

\section{References}

1. H. Tawancy, L. M. Al-Hadhrami, and F. Al-Yousef, Case Stud. Eng. Fail. Anal., 1, 6 (2013)

2. R. Ben-Mansour, M. Habib, A. Khalifa, K. Youcef-Toumi, and D. Chatzigeorgiou, Comput. Fluids, 57,110(2012)

3. C. Bersani, L. Citro, R. V. Gagliardi, R. Sacile, and A. M. Tomasoni, Chem. Engineer Trans, 19, 249 (2010)

4. M. Bertolini and M. Bevilacqua, JQME., 12,186 (2006)

5. M. Singh and T. Markeset, Eng. Fail. Anal.,16,2098 (2009)

6. M. Ahammed, Int. J. Press. Vessels Pip, 75,321 (1998)

7. M. S. El-Abbasy, A. Senouci, T. Zayed, and F. Mosleh, Struct. Infrastruct. Eng., 11, 263 (2015)

8. M. S. El-Abbasy, A. Senouci, T. Zayed, F. Mirahadi, and L. Parvizsedghy, J. Constr. Eng. Manage., 140, 04014013 (2014)

9. K. Mollazade, M. Omid, and A. Arefi, Comput. Electron. Agric., 84, 124 (2012) 